CLINICAL STUDY

\title{
Pregnancy in acromegaly: a one-center experience
}

\author{
Renato Cozzi, Roberto Attanasio and Michela Barausse \\ Division of Endocrinology, Ospedale Niguarda, Milan, Italy \\ (Correspondence should be addressed to R Cozzi; Email: renatocozzi@tiscali.it)
}

(R Attanasio is now at Division of Endocrinology, Ospedali Riuniti, Bergamo, Italy)

\begin{abstract}
Objective and design: The aim of the study was the retrospective evaluation of pregnancy in acromegalic women attending our center.

Patients and methods: Six active acromegalic women (30-35-years old, disease duration 5-17 years) underwent seven pregnancies. Four patients had macroadenoma and two microadenoma; four had surgery; and two had been treated primarily with drugs. Before conception, GH and IGF-I were 5.4 \pm 0.8 and $430 \pm 58 \mu \mathrm{g} / \mathrm{l}$ respectively. $\mathrm{GH}$ (by an assay unable to distinguish pituitary hormone from placental variant), IGF-I, and prolactin (PRL) levels were assessed before conception, every 3 months, and after delivery; visual field and magnetic resonance imaging were performed before delivery in the only patient with macroadenoma not previously operated on and after delivery in all.

Results: All the women conceived normally, after discontinuation of medications in five cases and, while on treatment with depot somatostatin analogs in two (discontinued after confirmation of pregnancy). All patients remained off-treatment throughout pregnancy, had uneventful pregnancies, and term delivery. The babies were healthy and normal in length and weight. Breast-feeding was allowed in four cases. During pregnancy, GH levels showed variable changes; IGF-I, notwithstanding the withdrawal of any GH hypersecretion-suppressive treatment, remained close to normal limits in all subjects and returned to pathological levels after delivery; PRL increased physiologically, returning to baseline level after delivery. In one of the two patients primarily treated with drugs, GH levels increased and the tumor regrew throughout pregnancy, although without visual impairment.

Conclusions: Pregnancy in acromegalic women has a normal course leading to a normal delivery, and produces normal babies. GH levels show variable changes, but decrease in most patients. IGF-I levels remain normal without medical treatment.
\end{abstract}

European Journal of Endocrinology 155 279-284

\section{Introduction}

Fertility is commonly impaired in patients with pituitary tumors due to hormonal hypersecretion, when functional, or mass effect causing destruction of gonadotropin-secreting cells or compression of both normal gonadotrope cells and pituitary stalk, producing hyperprolactinemia and anovulation. Moreover, excess adrenal androgen production also contributes to infertility in acromegaly $(1,2)$. Only limited data are available about pregnancy in acromegaly, although it appears that, after spontaneous or induced conception, pregnancy usually carries to term. However, it has been reported that growth hormone (GH)-secreting tumors may exhibit symptomatic enlargement during pregnancy (1), probably due to the growth-promoting effect of estrogens. Moreover, it is still debated whether ongoing GH-suppressive treatment should be maintained throughout pregnancy. The aim of this study was to review our experience about pregnancy in acromegalic women.

\section{Patients and methods}

Seven pregnancies (carried on between 2000 and 2002) in six acromegalic women (30-35-years old, disease duration 5-17 years) were retrieved from files and analyzed retrospectively. The study period evaluated in files begins after the introduction in our laboratory of an ultrasensitive GH assay. All women had active disease according to elevated GH levels not suppressible below $1 \mu \mathrm{g} / \mathrm{l}$ after oral glucose load and high age-adjusted insulin-like growth factor-I (IGF-I) levels. Four patients had macroadenoma and two, microadenoma. Serum prolactin (PRL) levels were pathological in five patients. Four patients had been previously submitted to non-curative neurosurgery (2-14 years before) and one had also been irradiated 14 years before; the remaining two had been primarily treated with drugs: the former with bromocriptine and latter with somatostatin analogs (SA). Thyroid and adrenal functions were normal in all patients. Clinical 
data are shown in Table 1. Informed consent of the patients was obtained for disclosing clinical investigation. GH, IGF-I, and PRL levels had been assessed before conception, and were monitored during pregnancy, every 3 months (at the end of first, second, and third trimester), and after delivery (at 2-month interval starting after 1 month). Visual field examination and magnetic resonance imaging (MRI) had been checked at 1 month after delivery in all patients, and also before delivery in patient no. 5, and compared to the last examination performed in the year before pregnancy while on GH-suppressive treatment. Each MRI had been regarded as unchanged in comparison to the previous examination.

At each control, blood samples had been collected in the morning at hourly intervals for at least $3 \mathrm{~h}$ after an overnight fast and rest, while the patients were supine and awake, with an indwelling needle inserted in an antecubital vein and kept patent by slow infusion of saline. GH and PRL concentrations were assayed on each sample (in Results, the reported value is the mean of all the samples) and IGF-I levels on the first sample. GH (DPC, Los Angeles, CA, USA), PRL (ADVIA Centaur, Bayer), and IGF-I (Nichols, San Juan de Capistrano, CA, USA) had been assayed in duplicate by immunometry, immunochemiluminescence, and chemiluminescence after acid-ethanol extraction respectively. GH-detection limit was $0.01 \mu \mathrm{g} / \mathrm{l}$. GH assay was unable to distinguish the pituitary hormone from the placental variant. Standards were calibrated against first IRP 80/505 ( $1 \mathrm{ng}=2.6 \mu \mathrm{IU}$ ) for $\mathrm{GH}$, third RP WHO 84/500 for PRL $(1 \mu \mathrm{g}=21.2 \mathrm{IU})$, and WHO 87/518 for IGF-I. Intra- and interassay coefficients of variation were $2.9-4.6$ and 4.2-6.6\% for GH, 3.2 and $4 \%$ for PRL, 3.7 and $7.2 \%$ for IGF-I. Normal values for PRL in females are $2.8-29.2 \mu \mathrm{g} / \mathrm{l}$, and for IGF-I in this age group, 115-307 $\mu \mathrm{g} / \mathrm{l}$. Values are expressed as mean \pm s.e.M. unless otherwise stated.

\section{Results}

\section{The mothers}

All the women conceived normally. Pregnancy started 3-4 months after discontinuation of medication in five cases and, while on treatment with depot SA in two (discontinued after confirmation of pregnancy; Table 2). During pregnancy, no patient complained of any symptom linked to acromegalic disease and clinical score was near normal. All patients had uneventful pregnancies. No patient developed either gestational diabetes, according to serum glucose levels evaluated before and during oral glucose tolerance test (OGTT) (at 60 and $120 \mathrm{~min}$ after $75 \mathrm{~g}$ ) performed at the 24th week, hypertension or pre-eclampsia. Delivery was at term in all, by the vaginal route in four and with programmed cesarean section in three.

\section{The babies}

At examination by neonatologist, the newborns (five males, two females) were normal in length and weight and no malformations or metabolic imbalances were found (in particular of glucose, calcium, and bilirubin). The regular follow-up did not disclose any growth impairment or disease. Breast-feeding was allowed in four cases and lactation was inhibited in one (no. 5) by cabergoline administration. In the other two cases, the mothers did not want to breast-feed or did not have lactation onset (nos 1 and $4 \mathrm{~b}$ respectively).

\section{The acromegalic disease}

At conception, GH and IGF-I levels were $5.4 \pm 0.8$ and $430 \pm 58 \mu \mathrm{g} / \mathrm{l}(140 \pm 50 \%$ upper limit of normal range) respectively. During pregnancy (Fig. 1), GH levels decreased by $50-90 \%$ in three women (nos 2, 3, and $4 \mathrm{a}$ ), remained stable in three (nos 1, 4b, and 6) and increased only in patient no. 5. IGF-I levels did not increase throughout pregnancy in any patient and remained near normal limits in all. PRL increased physiologically. After delivery, GH levels increased in 1-5 months, IGF-I returned pathological, and PRL to before pregnancy values. GH-suppressive treatment was started again in all, within 5 months. Visual fields remained normal in all patients. At 1 month after delivery, MRI control (Table 3) did not show any difference in comparison to the last examination performed before pregnancy in all but one patient

Table 1 Clinical data.

\begin{tabular}{|c|c|c|c|c|c|c|}
\hline \multirow[b]{2}{*}{ No. } & \multicolumn{2}{|c|}{ At diagnosis } & \multicolumn{2}{|c|}{ Previous treatments } & \multicolumn{2}{|c|}{ After NS ${ }^{a}$} \\
\hline & $\mathrm{GH}^{\mathrm{b}}$ & $\mathrm{PRL}^{\mathrm{b}}$ & $N S^{a}$ & $R T^{\mathrm{c}}$ & $\begin{array}{l}\text { Post-oral glucose } \\
\text { tolerance test } \mathrm{GH}^{\mathrm{b}}\end{array}$ & IGF-I ${ }^{b}$ \\
\hline 1 & 200 & 29 & 1986 & 1986 & 5.4 & 601 \\
\hline 2 & 50 & 32 & 2000 & - & 8 & 676 \\
\hline 3 & 40 & 80 & 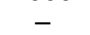 & - & - & - \\
\hline 4 & 60 & 28 & 1997 & - & 13 & 945 \\
\hline 5 & 150 & 50 & - & - & - & - \\
\hline 6 & 35 & 60 & 1996 & - & 6 & 363 \\
\hline
\end{tabular}

${ }^{\mathrm{a}}$ Neurosurgery (year). ${ }^{\mathrm{b}}$ In microgram/liter. ${ }^{\mathrm{c}}$ Radiotherapy (year). 
Table 2 Data about pregnancies.

\begin{tabular}{|c|c|c|c|c|c|c|c|c|c|}
\hline \multirow[b]{2}{*}{ No. } & \multicolumn{5}{|c|}{ At conception } & \multicolumn{4}{|c|}{ Newborns } \\
\hline & Year & Age & Drugs $^{a}$ & $\mathrm{GH}^{\mathrm{b}}$ & $\mid G F-I^{b}$ & Delivery & Sex & $\begin{array}{c}\text { Weight }^{\mathrm{c}} \\
\text { (percentile) }\end{array}$ & Breast-feeding \\
\hline 1 & 2000 & 31 & $\mathrm{Br}$, scOC: off & 2.6 & 311 & Vaginal at term & $\mathrm{F}$ & $3.0\left(15^{\circ}\right)$ & No \\
\hline 2 & 2002 & 30 & LAR, ATG: off & 7.5 & 676 & Cesarean at term & $M$ & $3.7\left(50^{\circ}\right)$ & Yes \\
\hline 3 & 2002 & 35 & Br, Quin: off & 5 & 414 & Vaginal at term & $\mathrm{M}$ & $3.7\left(50^{\circ}\right)$ & Yes \\
\hline $4 a$ & 2000 & 33 & LAR: on & 8.6 & 301 & Vaginal at term & M & $3.5\left(30^{\circ}\right)$ & No \\
\hline $4 \mathrm{~b}$ & 2002 & 35 & LAR: off & 3.1 & 328 & Vaginal at term & $\mathrm{M}$ & $4.3\left(80^{\circ}\right)$ & Yes \\
\hline 5 & 2002 & 35 & Lan: off; LAR: on & 6.2 & 615 & Cesarean at term & $\mathrm{M}$ & $3.2\left(15^{\circ}\right)$ & Inhibited \\
\hline 6 & 2002 & 32 & $\mathrm{Br}, \mathrm{Cab}$ : off & 4.9 & 363 & Cesarean at term & $\mathrm{F}$ & $3.9\left(90^{\circ}\right)$ & Yes \\
\hline
\end{tabular}

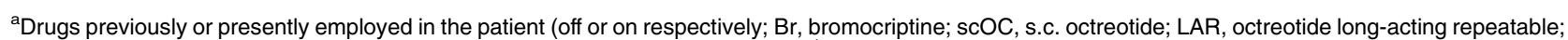

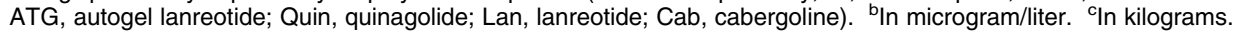

(no. 5). In patient no. 5, who, notwithstanding very high basal GH levels, had refused neurosurgery fearing pituitary damage, primary treatment with SA restored regular menses, achieved both an impressive decrease in GH/IGF-I levels, and the marked shrinkage of her huge macroadenoma. After SA withdrawal, GH progressively re-increased up to pre-treatment levels throughout pregnancy, but IGF-I did not change. Before delivery, MRI control showed a marked re-increase of tumor mass; however, neither symptoms nor ophthalmologic impairment occurred. SA treatment was started again at the first post-delivery control and neurosurgery was suggested thereafter.

\section{Discussion}

It is well known that pituitary tumors have profound effects on fertility, whereas pregnancy affects the behavior of pituitary tumors (1-3).

Even though fertility is commonly impaired in acromegaly, owing to frequent concomitant hyperprolactinemia and androgen excess due to GH hypersecretion, reproductive potential is preserved if tumor mass or ablative treatments did not previously destroy the gonadotropin lineage. Accordingly, spontaneous, as well as induced, conceptions were already reported (4-6), while both off- and on-treatment with GH-suppressing drugs, mostly with dopamine-agonist (6-8) but also with SA (9-11). All patients in this series conceived spontaneously, most while off-treatment and two while on-treatment with SA. It has been reported that if pregnancy is commenced, it is thereafter carried on until term without troubles in most reported cases (1), as in this series. In the largest series reported in abstract form up to date (6), one miscarriage was reported out of 31 pregnancies. Also, delivery is not different from that in women unaffected by pituitary diseases. As for babies, pre- and postnatal development is commonly reported to be absolutely normal $(1,6)$. It is common politics (1) to stop any GH-suppressive treatment, as we did, after confirmation of pregnancy, even though cases were reported in whom medications were continued throughout pregnancy. While bromocriptine is regarded

as safe even during pregnancy, due to its widespread use in patients affected by prolactinoma (12), data in pregnancy are still scarce about SA that became in recent years the mainstay of medical treatment in

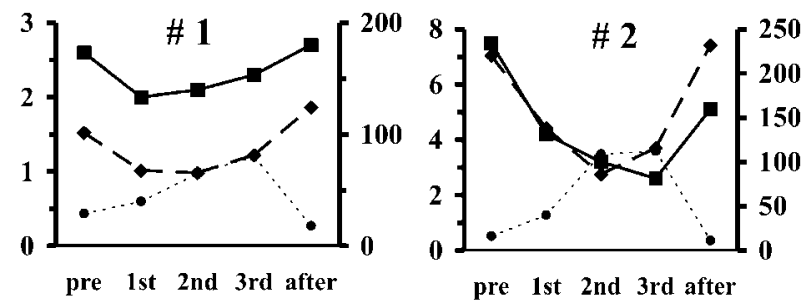

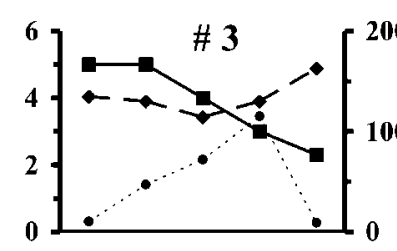

pre 1st 2nd 3rd after

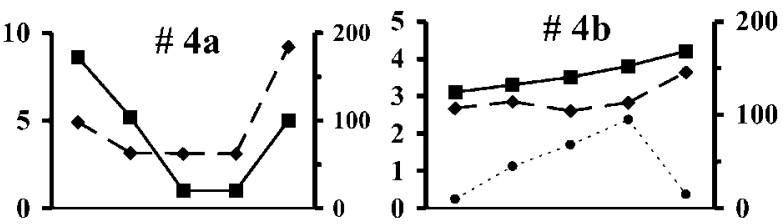

pre 1st 2nd 3rd after

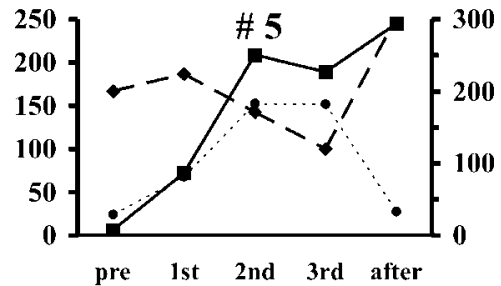

Figure 1 Growth hormone (expressed as microgram/liter with squares and continuous line on the left axis), insulin-like growth factor-I (expressed as percentage of the upper limit of normal range with diamonds and broken line on the right axis), and prolactin levels (expressed as microgram/liter with circles and dotted line on the right axis) before pregnancy, during the first, second, and third trimester, and 1-5 months after delivery are depicted for each pregnancy (ID as in Table 2 in the upper part of each panel). Please note that scale is different in different panels. 
Table 3 Tumor data (in brackets, volume in $\mathrm{mm}^{3}$, calculated as the volume of a rotating ellipsoid, with the formula: volume $=\pi($ coronal diameter $\times$ sagittal diameter $\times$ transverse diameter $) / 6(35))$.

\begin{tabular}{lllr}
\hline No. & Basal evaluation $^{\mathrm{a}}$ & Before pregnancy $^{\mathrm{b}}$ & After delivery $^{\mathrm{c}}$ \\
\hline 1 & Invasive intra-suprasellar macroadenoma (2724) & Cavernous and perichiasmatic remnant (69) & 59 \\
2 & Intra-suprasellar macroadenoma (1837) & Remnant (167) & 128 \\
3 & Intra-infrasellar macroadenoma (1256) & Shrunk adenoma (167) & 180 \\
$4 \mathrm{a}$ & Intra-suprasellar macroadenoma (1437) & Cavernous remnant (360) & 419 \\
$4 \mathrm{~b}$ & & Cavernous remnant (268) & 235 \\
5 & Intra-suprasellar macroadenoma (2969) & Empty sella (157) & 2094 \\
6 & Intra-sellar macroadenoma (1144) & Empty sella (9) & 9 \\
\hline
\end{tabular}

${ }^{a}$ At diagnosis. ${ }^{b}$ In the year before pregnancy (while on $\mathrm{GH}$-suppressive treatment). ${ }^{\mathrm{c}}$ At 1 month after delivery.

acromegaly. Theoretical concern about SA is multifaceted. It was demonstrated that these drugs are able to cross the placental barrier $(13,14)$. Somatostatin receptors are expressed during fetal life (15), and the mechanism of action of SA involves the suppression of growth factors, whose role in fetus development is not yet fully understood, but is certainly crucial. However, it has been suggested previously that somatostatin receptors in the newborn might not be immediately functional (16), and that octreotide administered in pharmacological doses does not bind to placental receptors (17). Most previously reported cases were treated with octreotide only up to pregnancy confirmation and only with the s.c. short-acting analog (18-22). In a few cases, octreotide was used for a longer period, i.e. for 6 months (23) or throughout the second and third trimester (10), or even throughout the whole gestation $(14,24)$. All newborns showed no malformations and normal postnatal development. With the use of long-acting formulations, the exposition of fetus, to SA is prolonged, even if the drug is withdrawn after pregnancy confirmation. It was reported that ultrasound monitoring of fetal parameters during octreotide long-acting repeatable treatment in a patient suggested the possibility of fetal growth retardation, prompting drug dose decrease (14). Thereafter, no further growth retardation was shown and postnatal development was reported as normal up to 18 months.

But what about the effect of pregnancy on hormone secretion and tumor size in acromegaly? In normal pregnancy, the GH axis is shifted from pituitary GH to its placental variant, that is the product of the $G H-V$ gene specifically expressed in the syncytio-trophoblast layer of the human placenta. Placental $\mathrm{GH}$ differs from pituitary GH by 13 amino acids. It has high somatogenic and low lactogenic activities and is secreted by the placenta in a non-pulsatile manner. Assays by specific monoclonal antibodies reveal that in the maternal circulation placental GH gradually replaces since 1520 weeks up to term pituitary $\mathrm{GH}$, which becomes undetectable (25). In addition, the increase of placental GH causes the augmentation of IGF-I levels, observed in normal pregnancies. In patients with acromegaly, pituitary GH hypersecretion is believed to remain autonomous, so that both GH levels should remain elevated during the entire pregnancy, at variance with normal women (22), and IGF-I levels further increase. Our results are in contrast with present beliefs. In fact, GH levels decreased in all but one patient throughout pregnancy. Our assay was not able to distinguish pituitary GH secretion from the placental one. It can be hypothesized that pituitary GH secretion in our patients was not entirely autonomous, but that, on the contrary, it was at least partly sensitive to the negative feedback control exerted by placental GH. This was already suggested by Obuobie et al. (26) in a case report of pregnant acromegalic woman with McCune-Albright syndrome. No IGF-I increase occurred in any patient throughout pregnancy, notwithstanding the withdrawal of any GH-suppressive treatment. Even though disease was mild in most, it was still active, as shown by pathologic IGF-I levels in all patients after delivery, prompting the reinstitution of GH-suppressive treatment. At the beginning of pregnancy, a probable carryover effect of previous SA treatment could have maintained IGF-I values close to normal range in the two patients still on treatment. This mechanism, however, could not account for IGF-I values in the other off-treatment patients, and in late pregnancy. It was reported that serum IGF-I increase in late pregnancy of acromegalic women was similar to that observed in normal pregnancy $(11,27-29)$. Beckers et al. (27) interpreted those findings as a confirmation that increased IGF-I levels are not pituitary GH-dependent in late pregnancy, whereas Aso et al. (28) suggested that the GH secreted in increasingly large amounts by the residual tumor mass during pregnancy was defective in certain biological properties. On the other hand, McIntyre et al. (30) showed that, although both total and free placental GH concentrations doubled between the 28th and the 36th week in normal women, IGF-I concentrations did not change significantly. In agreement with those suggestions, IGF-I and GH patterns were clearly discordant in patient no. 5 of this series, the only patient in whom we observed during pregnancy a clear-cut increase in GH levels but a trend towards decreasing IGF-I levels. Neither estrogen levels, nor human chorionic gonadotropin, or IGF-binding protein (IGF-BP) 1 should be different between normal and acromegalic women during pregnancy. As for IGF-BP3, 
that is GH-dependent, its levels should have been increased in the patient following $\mathrm{GH}$ increase and, in turn, should have induced IGF-I increase and not decrease, as it was actually observed. It was previously demonstrated (31) that IGF-BP3 proteolytic activity may be increased in pregnant serum, thus putatively increasing IGF-I bioavailability, but, as suggested by Baxter (32), even if an accelerated release of IGFs does occur, it is unclear whether the released IGFs would have increased availability to tissue receptors (local IGF-BPs might rapidly re-bind them), or would simply be lost through degradative clearance. Furthermore, during pregnancy, a shift to less bioactive $\mathrm{GH}$ isoforms, as well as a contribution of changes in GH-binding protein cannot be ruled out (30). Another possibility in some patients might be that the decrease in IGF-I levels could depend on the observed GH decrease.

The volume of pituitary gland enlarges during normal pregnancy, due to hyperplasia of mature lactotrophs, and pituitary size may increase by $45 \%$ during the first trimester (33). Theoretically, the stimulatory effect of peripheral hormone surges, such as estrogens, during pregnancy could cause adenoma enlargement due to tumor growth or hemorrhage, or tumor infarction in patients with GH-secreting adenomas (1). It has been reported in the series by Kupersmith et al. (34) that six out of eight women with macroadenoma (one out of two GH-secreting) developed visual field loss during pregnancy, and Okada et al. (29) described a patient, whose tumor size increased during pregnancy. In our series, MRI showed tumor expansion only in the patient with a clear-cut increase in GH levels during pregnancy, bearing the greatest sized adenoma. In that patient, tumor volume increase might likely be due to SA withdrawal with re-expansion of the tumor up to pretreatment size. It therefore seems appropriate to maintain a high level of control, at least in patients with large adenoma before treatment start.

Even though breast-feeding might represent a theoretical problem, due to the growth-promoting effect of suction dynamics on normal pituitary that may cause concern in patients with macroadenoma, all patients but the one with macroadenoma breast-fed and no problem was reported during this period.

As for glucose metabolism, GH is a powerful insulin antagonist, and pregnant acromegalic patients are prone to added glucose intolerance and diabetes. In all our patients, glucose was not found in serial urine dipstick routinely evaluated throughout pregnancy, newborn weight at birth was normal, as well as postdelivery serum glucose levels. During routine monitoring of pregnancy, no patient developed gestational diabetes, hypertension, or pre-eclampsia.

In conclusion, in our experience, pregnancy in acromegalic women yields normal babies. It has normal course and delivery, even without GH-suppressive treatment. GH levels show variable changes and in most patients decrease. IGF-I levels remain near normal throughout pregnancy without any medical treatment.

At present, even though clear evidence is still lacking, according to our limited experience as well as that of others (1), reasonable recommendations in women with active acromegaly may be as follows: before pregnancy occurs, control GH/IGF-I hypersecretion; withdraw long-acting SA 2 months before a planned pregnancy and any medical therapy when pregnancy is confirmed; check carefully patients with macroadenoma not previously submitted to ablative treatment, monitoring visual fields, reserving MRI studies only for those with a clear-cut re-increase of GH levels; in patients previously treated by non-curative neurosurgery, GH-suppressive treatment is not needed and spontaneous delivery can be safely carried out; in patients treated primarily only with drugs, carrying on medical treatment in pregnancy may be suggested with caution (due to wide distribution of somatostatin receptors in the fetus, including the brain) in those patients with previous large macroadenoma and evidence of tumor enlargement; in these patients, a tight ophthalmologic followup is mandatory and cesarean section is strongly suggested, to avoid that endocranic pressure increase during labor could induce pituitary apoplexy; breastfeeding can be safely allowed in patients with tumor remnant. A close collaboration between endocrinologist, obstetrician, and pediatrician is, of course, mandatory.

\section{References}

1 Herman-Bonert V, Seliverstov M \& Melmed S. Pregnancy in acromegaly: successful therapeutic outcome. Journal of Clinical Endocrinology and Metabolism 199883 727-731.

2 Frohman L. Pituitary tumors in pregnancy. Endocrinologist 2001 11 399-406.

3 Molitch ME. Pituitary tumors and pregnancy. GH and IGF Research 200313 S38-S44.

4 Kretz S, Ledoyen S, Kahal Z, Vesco L, Bernheim R, Keravel Y, Kujas M, Brugieres M \& Perlemuter L. Pregnancy after in vitro fertilization in an acromegalic woman. (abstract) Fertility and Sterility 199870 (Suppl 1) 214.

5 Esfandiari N, Gotlieb L \& Casper RF. Live birth of healthy triplets after in vitro fertilization and embryo transfer in an acromegalic woman with elevated growth hormone. Fertility and Sterility 2005 831041.

6 Caron P, Bertherat J, Borson-Chazot F, Brue T, Cortet-Rudelli C \& Chanson P. Outcome of 43 pregnancies in acromegalic women. Proceedings of 87th Annual Meeting of Endocrine Society, 2005 P3-P552.

7 Luboshitzky R, Dickstein G \& Barzilai D. Bromocriptine-induced pregnancy in an acromegalic patient. Journal of the American Medical Association $1980 \mathbf{2 4 4} 584-586$.

8 Miyakawa I, Taniyama K, Koike H, Mori N, Nagamine M, Kuribayashi T \& Araki S. Successful pregnancy in an acromegalic patient during 2-Br-alpha-ergocryptine (CB-154) therapy. Acta Endocrinologica $1982 \mathbf{1 0 1} 333-338$.

9 de Menis E, Billeci D, Marton E \& Gussoni G. Uneventful pregnancy in an acromegalic patient treated with slow-release lanreotide: a case report. Journal of Clinical Endocrinology and Metabolism 1998 $83727-731$. 
10 Takeuchi K, Funakoshi T, Oomori S \& Maruo T. Successful pregnancy in an acromegalic women treated with octreotide. Obstetrics and Gynecology 199993848.

11 Serri O \& Lanoie G. Successful pregnancy in a woman with acromegaly treated with octreotide long-acting release. Endocrinologist 200313 17-19.

12 Turkalj I, Braun P \& Krupp P. Surveillance of bromocriptine in pregnancy. JAMA 1982247 1589-1591.

13 Caron P, Gerbeau C \& Pradayrol L. Maternal-fetal transfer of octreotide. New England Journal of Medicine 1995333 601-602.

14 Fassnacht M, Capeller B, Arlt W, Steck T \& Allolio B. Octreotide LAR treatment throughout pregnancy in an acromegalic woman. Clinical Endocrinology 200155 411-415.

15 Epelbaum J. Multiple somatostatin functions along brain development and aging. Journal of Endocrinological Investigations 19972011.

16 Caron P, Bennet A, Lorenzini F, Gerbeau C, Igout A, Fauvel J,Tauber M \& Fournie A. Normal pregnancy in a woman with nesidioblastosis treated with somatostatin analog octreotide. In Program and Abstracts of the 81st Annual Meeting of the Endocrine Society, 1999 San Diego.

17 Caron P, Buscail L, Beckers A, Esteve JP, Igout A, Hennen G \& Susini C. Expression of somatostatin receptor SST4 in human placenta and absence of octreotide effect on human placental growth hormone concentration during pregnancy. Journal of Clinical Endocrinology and Metabolism 199782 3771-3776.

18 Landolt AM, Schmid J, Wimpfheimer C, Karlsson E \& Boerlin V. Successful pregnancy in a previously infertile woman treated with SMS 201-995 for acromegaly. New England Journal of Medicine $1989320671-672$.

19 Montini M, Pagani G, Gianola D, Pagani MD, Piolini R \& Camboni MG. Acromegaly and primary amenorrhea: ovulation and pregnancy induced by SMS 201-995 and bromocriptine. Journal of Endocrinological Investigations 199013193.

20 Paiva I, Barros L, Gomes F, Fagulha A, Fagulha I, Carvalheiro M \& Ruas MMA. Successful pregnancy in an acromegalic woman treated with octreotide. IV European Congress of Endocrinology, Sevilla, Spain, (1998) Abstract, P2-P99.

21 Hierl T, Ziegler R \& Kasperk C. Pregnancy in persistent acromegaly. Clinical Endocrinology 200053 262-263.

22 Neal JM. Successful pregnancy in a woman with acromegaly treated with octreotide. Endocrine Practice 20006 148-150.

23 Mozas J, Ocon E, Lopez de la Torre M, Suarez AM, Miranda JA \& Herruzo AJ. Successful pregnancy in a woman with acromegaly treated with somatostatin analog (octreotide) prior to surgical resection. International Journal of Gynaecology and Obstetrics 1999 $6571-73$.

24 Mikhail N. Octreotide treatment of acromegaly during pregnancy. Mayo Clinic Proceedings 200277 297-298.
25 Alsat E, Guibourdenche J, Luton D, Frankenne F \& Evain-Brion D. Human placental growth hormone. American Journal of Obstetrics and Gynecology $19971771526-1534$.

26 Obuobie K, Mullik V, Jones C, John R, Rees AE, Davies JS, Scanlon MF \& Lazarus JH. McCune-Albright syndrome: growth hormone dynamics in pregnancy. Journal of Clinical Endocrinology and Metabolism 200186 2456-2458.

27 Beckers A, Stevenaert A, Foidart JM, Hennen G \& Frankenne F. Placental and pituitary growth hormone secretion during pregnancy in acromegalic women. Journal of Clinical Endocrinology and Metabolism 199071 725-731.

28 Aso T, Goto K, Takeuchi J, Kotsuji F \& Tominaga T. A triplet pregnancy after gonadotropin-releasing hormone pulsatile infusion therapy in a postoperative case of growth hormoneproducing pituitary macroadenoma. Endocrine Journal 198734 395-405.

29 Okada Y, Morimoto I, Ejima K, Yoshida K, Kashimura M, Fujihira T $\&$ Eto $S$. A case of active acromegalic woman with a marked increase in serum insulin-like growth factor-1 levels after delivery. Endocrine Journal 199744 117-120.

30 McIntyre HD, Serek R, Crane DI, Veveris-Lowe T, Parry A, Johnson S, Leung KC, Ho KK, Bougoussa M, Hennen G, Igout A, Chan FY, Cowley D, Cotterill A \& Barnard R. Placental growth hormone (GH), $\mathrm{GH}$-binding protein, and insulin-like growth factor axis in normal, growth-retarded, and diabetic pregnancies: correlations with feta growth. Journal of Clinical Endocrinology and Metabolism $2000 \mathbf{8 5}$ 1143-1150.

31 Lassarre C \& Binoux M. Insulin-like growth factor binding protein3 is functionally altered in pregnancy plasma. Endocrinology 1994 134 1254-1262.

32 Baxter RC. What is the significance of IGF-binding protein-3 proteolysis in the circulation? Journal of Clinical Endocrinology and Metabolism 2001865087.

33 Gonzalez J, Elizondo G, Saldivar D, Nanez H, Todd L \& Villareal J. Pituitary gland growth during normal pregnancy: an in vivo study using magnetic resonance imaging. American Journal of Medicine $198885217-220$.

34 Kupersmith MJ, Rosenberg C \& Kleinberg D. Visual loss in pregnant women with pituitary adenomas. Annals of Internal Medicine 1994121 473-477.

35 Di Chiro G \& Nelson KB. The volume of the sella turcica. American Journal of Radiology 1962 87 989-1008.

Received 24 January 2006

Accepted 24 May 2006 